\title{
Clinical Utility of a Plasma Protein Classifier for Indeterminate Lung Nodules
}

\author{
Anil Vachani ${ }^{1} \cdot$ Zane Hammoud $^{2} \cdot$ Steven Springmeyer $^{7} \cdot$ Neri Cohen $^{3}$. \\ Dao Nguyen ${ }^{4} \cdot$ Christina Williamson $^{5} \cdot$ Sandra Starnes $^{6} \cdot$ Stephen Hunsucker $^{7}$. \\ Scott Law ${ }^{7}$ Xiao-Jun $\mathrm{Li}^{7} \cdot$ Alexander Porter $^{7} \cdot$ Paul Kearney $^{7}$
}

Received: 21 June 2015/ Accepted: 7 September 2015/Published online: 16 September 2015

(C) The Author(s) 2015. This article is published with open access at Springerlink.com

\begin{abstract}
Evaluation of indeterminate pulmonary nodules is a complex challenge. Most are benign but frequently undergo invasive and costly procedures to rule out malignancy. A plasma protein classifier was developed that identifies likely benign nodules that can be triaged to CT surveillance to avoid unnecessary invasive procedures. The clinical utility of this classifier was assessed in a prospective-retrospective analysis of a study enrolling 475 patients with nodules $8-30 \mathrm{~mm}$ in diameter who had an invasive procedure to confirm diagnosis at 12 sites. Using this classifier, $32.0 \%$ (CI 19.5-46.7) of surgeries and $31.8 \%$ (CI 20.9-44.4) of invasive procedures (biopsy and/or surgery) on benign nodules could have been avoided. Patients with malignancy triaged to $\mathrm{CT}$ surveillance by the classifier would have been $24.0 \%$ (CI 19.2-29.4). This rate is similar to that described in clinical practices $(24.5 \% \mathrm{CI}$ 16.2-34.4). This study demonstrates the clinical utility of a non-invasive blood test for pulmonary nodules.
\end{abstract}

Clinical Trial Registration: Registered at ClinicalTrials.gov (NCT01752101).

Electronic supplementary material The online version of this article (doi:10.1007/s00408-015-9800-0) contains supplementary material, which is available to authorized users.

Paul Kearney

pkearney@indidx.com

1 Pulmonary, Allergy, and Critical Care Division, Perelman School of Medicine, University of Pennsylvania/Abramson Research Center, 3615 Civic Center Boulevard, Suite 1016E, Philadelphia, PA 19104, USA

2 Henry Ford Hospital, 2799 W. Grand Blvd., Detroit, MI 48202, USA

3 Greater Baltimore Medical Center, 6569 North Charles Street, Suite 701, Baltimore, MD 21204, USA
Keywords Xpresys lung · Lung nodule - Clinical utility · Prospective $\cdot$ Biomarker $\cdot$ Lung cancer

\section{Introduction}

A large number of pulmonary nodules are identified annually in the U.S. presenting a difficult clinical challenge, as the majority ultimately prove to be of benign origin [1]. Physicians are faced with developing a diagnostic strategy that identifies nodules that are malignant and yet minimizes the risks of invasive procedures on benign nodules. Currently available tools to assist physicians include risk prediction models [2] and imaging, such as PET/CT. However, evidence suggests that these tools have limitations in practice $[3,4]$.

With the adoption of low-dose CT (LDCT) screening for lung cancer [5] and the anticipated increase in pulmonary nodules, there is a growing interest in biomarkers as diagnostic adjuncts [6,7]. Advances in proteomic technologies permitted the development of a 371 protein classifier composed of lung cancer-associated proteins [6]. The discovery phase identified a subset of five diagnostic proteins that participate in several lung cancer-associated

4 Sylvester Comprehensive Cancer Center, University of Miami Hospital \& Clinics, 1550 NW 10th Avenue, Fox Building, Suite 308, Office 314, Miami, FL 33136, USA

5 Department of Thoracic and Cardiovascular Surgery, Lahey Hospital \& Medical Center, 41 Mall Road, Burlington, MA 01805, USA

6 University of Cincinnati, 231 Albert Sabin Way, Cincinnati, OH 45267-0562, USA

7 Integrated Diagnostics, 818 Stewart St., Suite 1101, Seattle, WA 98101, USA 
pathways (pathogenesis, chronic lung inflammation, oxidative stress response) [6]. The classifier was further validated in patients with indeterminate pulmonary nodules to identify likely benign nodules with high accuracy (negative predictive value $90 \%$, sensitivity $92 \%$ and specificity $20 \%$ ). The classifier is additive to currently used clinical risk factors, and has high analytic performance $[8,9]$. The intent of the present study is to evaluate the clinical utility of this classifier in a multicenter study of patients with indeterminate nodules undergoing invasive diagnostic procedures.

\section{Methods}

\section{Study Design and Population}

The study used a prospective-specimen-collection and retrospective-blinded-evaluation (prospective-retrospective) design. Patients with an indeterminate pulmonary nodule were enrolled at 12 geographically diverse sites in the U.S. Eligible patients were those with a lung nodule between 8 and $30 \mathrm{~mm}$ in diameter, minimum 40 years of age, and had recently completed a CT-guided needle aspiration (TTNA) or bronchoscopic biopsy with an established diagnosis or scheduled for a surgical lung biopsy. Exclusion criteria included a prior malignancy within 5 years of lung nodule identification or a clinical tumor stage $\geq \mathrm{T} 2$, nodal stage $\geq \mathrm{N} 2$, or evidence of metastatic disease.

All TTNA and bronchoscopy procedures were categorized as either diagnostic (provided a specific malignant or benign pathological diagnosis), or non-diagnostic (the specific etiology of the lung nodule remained unknown). All surgical procedures were categorized into either diagnostic (i.e., no specific prior diagnosis) or therapeutic (i.e., surgery preceded by a TTNA or bronchoscopy that yielded a malignant diagnosis).

For comparison to practice patterns, we utilized a retrospective-observational study of 377 patients with pulmonary nodules between 8 and $20 \mathrm{~mm}$, presenting to 18 geographically representative outpatient pulmonary clinics $[3,4]$. In addition to the published data for the study, an additional analysis was performed to determine the number of non-small cell lung cancer (NSCLC) patients triaged to CT surveillance during management, specifically, those having a second CT scan at least 3 months after the initial CT scan and no sooner than 3 days before surgery.

\section{Protein Expression Classifier Analysis}

Samples collected from subjects prior to definitive excision or treatment of the nodule were analyzed using multiple reaction monitoring as previously described $[6,8]$. The protein classifier, Xpresys ${ }^{\circledR}$ Lung, consists of 5 diagnostic and 6 normalization proteins and has been analytically and clinically validated $[6,8,9]$. The classifier reports "likely benign" when the probability of the lung nodule having benign etiology is high (NPV at least $84 \%$ ) and the remainder are reported as "indeterminate." In accordance with best practices, the classifier was adjusted for evaluation of archival specimens included in this study (see Supplementary). Physicians and patients were blinded to the protein classifier results and laboratory personnel blinded to clinical outcomes.

\section{Statistical Analysis}

To assess the ability of the classifier to improve the diagnostic evaluation of indeterminate lung nodules and its clinical utility, we assessed all procedures and outcomes and modeled the change in invasive procedures that would occur if the classifier results were used prospectively for decision making. The primary outcomes for this analysis were the potential benefits, i.e., reduction in invasive procedures (TTNA, bronchoscopy, and surgery), and the potential harms, i.e., rate of patients with malignant nodules routed to CT surveillance. This analysis assumes that all patients with a likely benign result would not undergo surgery or other invasive testing, but instead would be evaluated by CT surveillance.

Continuous and categorical variables were assessed using Mann-Whitney and Fisher's exact tests. Confidence intervals (CIs) are reported as two-sided binomial $95 \%$ CIs. Statistical analysis was performed using the R statistical package [10].

\section{Results}

A total of 475 subjects were enrolled prospectively from April 2012 to December 2014 in the registered study, NCT01752101. Of these, 353 patients were eligible for the clinical utility analysis; 287 (81.3\%, CI 76.8-85.2\%) were diagnosed with NSCLC, and 66 (18.7\%, CI 14.8-23.2\%) diagnosed with a benign lung nodule (Fig. 1). Reasons for subject ineligibility included violating the inclusion/exclusion requirements of the registered study (50 subjects), diagnosed with a cancer other than NSCLC (43 subjects), unable to obtain a definitive diagnosis due to missing data (26 subjects) and improper sample handling resulting in unanalyzable sample (3 subjects). Baseline demographics are shown in Table 1. Lung cancer patients were older ( $p$ value 0.03 ), and had greater cumulative tobacco packyears ( $p$ value 0.04$)$. No difference in nodule size between groups was observed ( $p$ value 0.45 ). 
Fig. 1 Flowchart of subjects included in clinical utility analyses along with categorization of subjects by procedure, outcome and classifier report. 'LB' and 'IND' represent a classifier 'Likely Benign' and 'Indeterminate' report, respectively. Dx indicates diagnostic, Non-Dx is a non-diagnostic procedure, and Dx Biopsy includes patients with a biopsy only and those that had a diagnostic biopsy followed by surgery

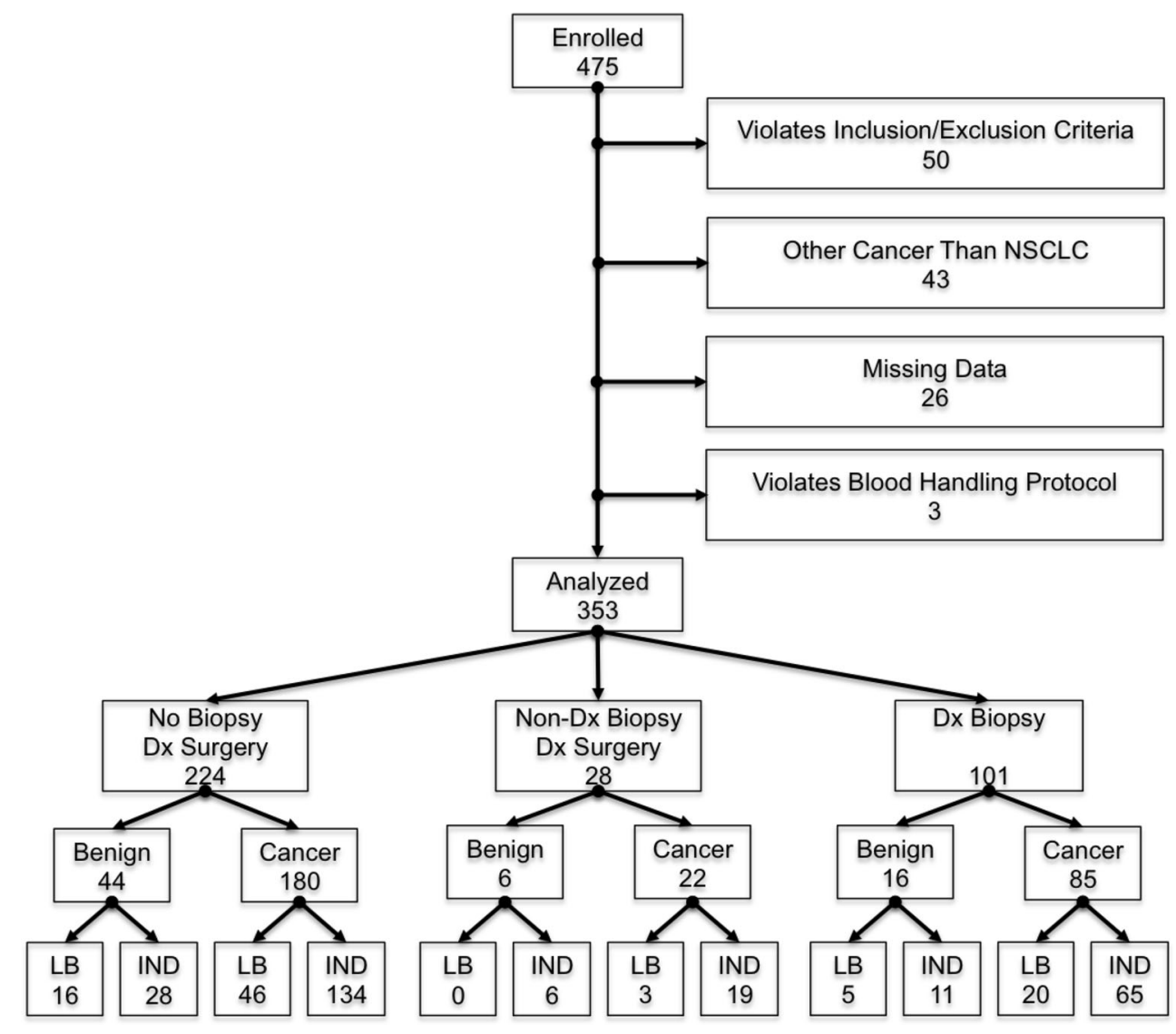

Table 1 Patient demographics stratified by diagnosis

\begin{tabular}{lllll}
\hline Characteristics & All patients & Cancer & Benign & $p$ value \\
\hline Patients & 353 & 287 & 66 & \\
Age (years) [mean (range)] & $68(44.7-95.5)$ & $68.6(45.3-95.5)$ & $65.4(44.7-87.5)$ & $0.027^{\mathrm{a}}$ \\
Gender $(n, \%)$ & & & & $0.272^{\mathrm{b}}$ \\
$\quad$ Male & $154(43.6)$ & $121(42.2)$ & $33(50.0)$ & \\
Female & $199(56.4)$ & $166(57.8)$ & $33(50.0)$ & \\
Smoking history & & & & $0.220^{\mathrm{b}}$ \\
Status (n, \%) & $60(17.0)$ & $46(16.0)$ & $14(21.2)$ & $37(56.1)$ \\
$\quad$ Never & $210(59.5)$ & $173(60.2)$ & $12(18.2)$ & \\
$\quad$ Former & $76(21.5)$ & $64(22.3)$ & $3(4.5)$ & $0.044^{\mathrm{a}}$ \\
$\quad$ Current & $7(2.0)$ & $4(1.3)$ & $35.1(0.5-120)$ & \\
$\quad$ Passive exposure & $41.5(0.5-150)$ & $42.8(0.8-150)$ & & \\
$\quad$ Pack-year mean (range) & & & \\
Lung nodules & $18.5(8.0-30)$ & $18.6(8.0-30)$ & $18.0(8.0-30)$ & $0.447^{\mathrm{a}}$ \\
$\quad$ Size (mm) [mean (range)] & & & \\
\hline
\end{tabular}

${ }^{a}$ Based on Mann-Whitney test (cancer versus benign)

b Based on Fisher's exact test (cancer versus benign)

c Based on $n=285$ (benign $=48$ and cancer $=237$ )
The 353 patients were evaluated by procedure type (Fig. 1). A total of 101 patients were enrolled following a diagnostic TTNA or bronchoscopy biopsy, yielding 16 (15.8\%, CI 9.3-24.4\%) benign lesions and $85(84.2 \%$,
75.6-90.7\%) patients with NSCLC. A total of 252 patients underwent a surgical lung biopsy; 224 (88.9\%, CI 84.3-92.5\%) did not have a preceding biopsy and 28 (11.1\%, CI $7.5-15.7 \%)$ were performed following a 
biopsy that was non-diagnostic. Among the 224 patients proceeding directly to surgical lung biopsy, 44 had a benign diagnosis $(19.6 \%$, CI $14.7-25.5 \%)$ and $180 \mathrm{had}$ NSCLC (80.4 \%, CI 74.5-85.3\%). Among the 28 patients undergoing surgical lung biopsy following a non-diagnostic biopsy, 6 had a benign diagnosis $(21.4 \%$, CI 8.3-41\%) and 22 had NSCLC (78.6\%, CI 59-91.7\%).

In total, 66 of $353(18.7 \%$, CI 14.8-23.2\%) patients that underwent an invasive procedure (TTNA, bronchoscopy, surgical lung biopsy) were ultimately diagnosed with a benign nodule. This included 50 of $252(19.8 \%$, CI 15.1-25.3\%) patients that underwent a surgical lung biopsy and 22 of 129 (17.1\%, CI 11.0-24.7\%) patients that had a bronchoscopy or TTNA. Six $(9.1 \%$, CI 3.4-18.7\%) patients with benign lesions underwent multiple invasive procedures.

To estimate the effect of the classifier on the number of invasive procedures in patients with benign nodules, we determined the "likely benign" classifier result among patients. The classifier predicted the nodule to be likely benign in 16 of 50 patients (32.0\%. CI 19.5-46.7\%) determined to have a benign lesion by surgical lung biopsy. When considering all invasive procedures, the classifier predicted the nodule to be likely benign in 21 of 66 patients (31.8\%, CI 20.9-44.4\%) with benign nodules diagnosed by either TTNA, bronchoscopy biopsy, or surgical lung biopsy.

Of the 287 cancers identified in the study, the classifier predicted "likely benign" in 49 patients $(17.1 \%$, CI $12.9-21.9 \%)$ that were diagnosed by surgical biopsy and in 69 patients (24.0\%, CI 19.2-29.4\%) diagnosed by any invasive procedure. Consequently, 17-24\% of patients with a lung cancer nodule would be triaged to CT surveillance. This was similar to the rate observed in the retrospective-observational cohort where 23 of 94 patients (24.5\%, CI $16.2-34.4 \%)$ with nodules ultimately diagnosed as NSCLC underwent CT surveillance during nodule management.

\section{Discussion}

This study presents the clinical utility of a protein classifier for the management of indeterminate pulmonary nodules. Clinical utility is "the balance of benefits and harms associated with the use of the test in practice, including improvement in measureable clinical outcomes and the usefulness or added value in decision-making compared with not using the test" [11]. This prospective-retrospective study demonstrates that use of the classifier produces the potential benefit of reducing unnecessary surgeries $(32.0 \%)$ and invasive procedures $(31.8 \%)$. The potential harm (malignant lung nodules routed to CT surveillance) is
$17.1 \%$ if the classifier is used prior to surgery and $24.0 \%$ if used prior to any invasive procedure. This compares favorably to $24.5 \%$ as observed in 18 pulmonary clinic practices $[3,4]$. This indicates that this classifier can provide incremental clinical utility over usual care. Importantly, the level of evidence is high at level 1B [12] which compares favorably to the GRADE $1 \mathrm{C}$ and $2 \mathrm{C}$ recommendations within the American College of Chest Physicians (ACCP) guidelines on pulmonary nodule management [13-15].

The risks and expense of biopsies and surgery are considerable with TTNA having pneumothorax rates of $15 \%$ and surgery mortality rates of $1-5 \%$ [13]. Notably, nearly $10 \%$ of patients in this study had multiple procedures. These rates of complications are of increasing concern as screening LDCT is now recommended for those at high risk of lung cancer. Thirty-nine percent of patients undergoing LDCT had a least one positive screen, with the majority (96\%) being false positives [16].

The strengths of this study include its magnitude, geographic diversity, high level of evidence, and generalizability. A limitation is the comparison of rates of malignant nodules routed to CT surveillance by usual care $(24.5 \%)$ to the classifier $(17.1 \% ; 24.0 \%)$. Although both estimates are taken over all malignant nodules, the former includes nodules between 8 and $20 \mathrm{~mm}$ and the present analysis includes nodules between 8 and $30 \mathrm{~mm}$ in diameter.

In summary, this classifier can assist physicians with the challenging task of differentiating benign and malignant pulmonary nodules for the purpose of reducing unnecessary invasive procedures on benign nodules. This classifier can be used early in the evaluation process, in combination with physician assessment, and before choosing an invasive procedure in the evaluation of newly discovered 8-30 mm pulmonary nodules (Fig. 2).

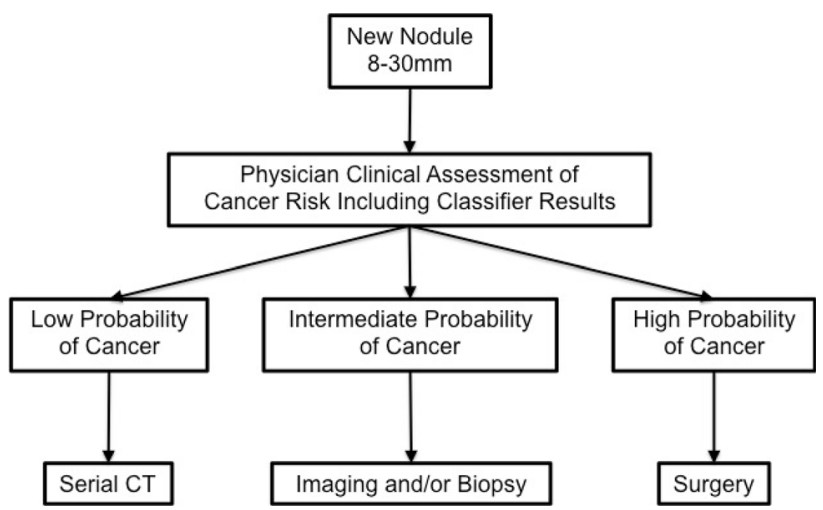

Fig. 2 The incorporation of the classifier into the ACCP guidelines for lung nodule management. Newly identified lung nodules between 8 and $30 \mathrm{~mm}$ in diameter are assessed using the plasma protein classifier. The classifier results are integrated into the physician's assessment of cancer risk 
Acknowledgments Authors wish to acknowledge the efforts of participating sites, analysts at Integrated Diagnostics and Ken Fang for insightful comments on the manuscript. Supported by Integrated Diagnostics

Author Contributions All authors made substantial contributions to the concept and design of the study; the acquisition, analysis or interpretation of the data; participated in the drafting and revision of the article critically for important intellectual content; and gave their final approval of the version to be published.

\section{Compliance with Ethical Standards}

Conflict of Interest Dr. Vachani has received consulting fees from Integrated Diagnostics. Dr. Springmeyer is employed by Integrated Diagnostics. Dr. Hunsucker is employed by Integrated Diagnostics. Dr. Law is employed by Integrated Diagnostics. Dr. Li is employed by Integrated Diagnostics. Dr. Porter is employed by Integrated Diagnostics. Dr. Kearney is employed by Integrated Diagnostics.

Ethical approval and Consent All procedures performed in studies involving human participants were in accordance with the ethical standards of the institutional and/or national research committee and with the 1964 Helsinki declaration and its later amendments or comparable ethical standards. Informed consent was obtained from all individual participants included in the study.

Open Access This article is distributed under the terms of the Creative Commons Attribution 4.0 International License (http://crea tivecommons.org/licenses/by/4.0/), which permits unrestricted use, distribution, and reproduction in any medium, provided you give appropriate credit to the original author(s) and the source, provide a link to the Creative Commons license, and indicate if changes were made.

\section{References}

1. Ost D, Fein AM, Feinsilver SH (2003) The solitary pulmonary nodule. N Engl J Med 348(25):2535-2542

2. Gould MK, Ananth L, Barnett PG (2007) A clinical model to estimate the pretest probability of lung cancer in patients with solitary pulmonary nodules. Chest 131:383-388

3. Tanner NT, Aggarwal J, Gould MK, Kearney P, Diette G, Vachani A, Fang KC, Silvestri GA (2015) Management of pulmonary nodules by community pulmonologists: a multicenter observational study. Chest. doi:10.1378/chest.15-0630

4. Vachani A, Tanner NT, Diette GB, Aggarwal J, Mathews C, Goss T, Kearney P, Fang K, Silvestri G (2014) A Markov model derived to quantify drivers of decision making in the evaluation of patients with pulmonary nodules. Am J Repir Crit Care Med 189:A2259

5. Jensen T, Chin J, Ashby L. Hermansen J, Hutter JD (2015) Decision memo for screening for lung cancer with low dose computed tomography (LDCT). http://www.cms.gov/medicarecoverage-database/details/nca-decision-memo.aspx?NCAId=274. Accessed 8 June 2015

6. Li XJ, Hayward C, Fong PY et al (2015) A blood-based proteomic classifier for the molecular characterization of pulmonary nodules. Sci Transl Med 5(207):207ra142

7. Spira A for the AEGIS Study Team, Silvestri GA, Vachani A, Whitney D, Elashoff M, Smith PK, Ferguson JS, Parsons E, Mitra N, Brody J, Lenburg ME (2015) A bronchial genomic classifier for the diagnostic evaluation of lung cancer. N Engl J Med 365:395-409

8. Vachani A, Pass H, Rom WN et al (2015) Validation of a multiprotein plasma classifier to identify benign lung nodules. J Thorac Oncol 10:629-637

9. Li XJ, Lee LW, Hayward C et al (2015) An integrated quantification method to increase the precision, robustness, and resolution of protein measurement in human plasma samples. Clin Proteomics 12:1-17

10. R Core Team (2013) R: a language and environment for statistical computing. R Foundation for Statistical Computing, Vienna, Austria. http://www.R-project.org/

11. Haddow J, Palomaki G (2003) ACCE: a model process for evaluating data on emerging genetic tests. In: Khoury M, Little J, Burke W (eds) Human genome epidemiology: a scientific foundation for using genetic information to improve health and prevent disease. Oxford University Press, Oxford, pp 217-233

12. Atkins D, Eccles M, Flottorp S et al (2004) Systems for grading the quality of evidence and the strength of recommendations I: critical appraisal of existing approaches The GRADE Working Group. BMC Health Serv Res 4(38):1-7

13. Gould MK, Donington J, Lynch WR et al (2013) Evaluation of individuals with pulmonary nodules: when is it lung cancer? Diagnosis and management of lung cancer, 3rd ed: American College of Chest Physicians evidence-based clinical practice guidelines. Chest 143:e93S-e120S

14. Simon R, Paik S, Hayes DF (2009) Use of archived specimens in evaluation of prognostic and predictive biomarkers. J Natl Cancer Inst 101:1446-1452

15. Hayes DF, Bast RC, Desch CE et al (1996) Tumor marker utility grading system: a framework to evaluate clinical utility of tumor markers. J Natl Cancer Inst 88(20):1456-1466

16. Aberle DR, Adams AM, Berg CD et al (2011) Reduced lung cancer mortality with low-dose computed tomographic screening. N Engl J Med 365:395-409 